\title{
Schwaches Herz: Fischöl wirksamer als Statin
}

\author{
Chefredakteur Dr. med. Dirk Einecke
}

\section{HERZINSUFFIZIENZ}

\section{Fischöl wirksam, Statin nutzlos}

Überraschende Ergebnisse brachte die italienische GISSI-Herzinsuffizienz-Studie: Die prüfte an etwa 7000 Patienten, ob die zusätzliche Gabe eines Statins oder von Kapseln mit Omega-3-Fettsäuren einen Zusatznutzen erwarten lässt. Was das Statin betrifft (das in Deutschland nicht erhältliche Rosuvastatin), so wurden die Hoffnungen enttäuscht: Weder die Mortalität noch die Morbidität war im Vergleich zur Kontrollgruppe verändert (29\% vs. $28 \%$ Todesfälle nach vier Jahren). Im Gegensatz dazu zeigte die Supplementierung mit Omega-3-Fettsäuren (1 g/d)

\section{EUROASPIRE III}

\section{In der Primärprävention gibt es noch viel zu tun}

Das Management von Hochrisikopatienten in der Primärprävention ist unzureichend, besagen die Ergebnisse der europäischen Studie EUROASPIRE III. Auch wenn bei diesen Patienten bereits eine antihypertensive, lipidsenkende oder antidiabetische Therapie eingeleitet ist, erreicht nur eine Minderheit die entsprechenden Zielwerte (Blutdruck 29\%, Cholesterin $31 \%$, Nüchtern-BZ $27 \%$ und $\mathrm{HbA}_{1 \mathrm{C}}$ $53 \%)$. Zudem sind vier von fünf Patienten übergewichtig oder adipös, 40\% machen keinen Sport und jeder Sechste raucht. Ein möglicher Grund: Die meisten Patienten sind über ihr gesundheitliches Risiko nicht aufgeklärt und unterschätzen es.

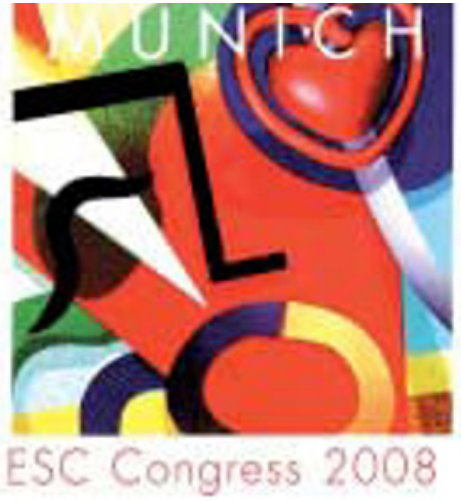

einen moderaten, aber zuverlässigen Nutzen. Die Gesamtmortalität wurde relativ um 9\% reduziert (27,3\% vs. 29,1\%).

\section{KHK-PATIENT MIT NIEDRIGEM LDL}

\section{Aggressive Lipidtherapie ohne Vorteil}

Je niedriger, desto besser: So lautet die oft zitierte Formel für die LDL-Cholesterin-Senkung bei Patienten in der Sekundärprävention. Einer erneuten Analyse der PROVE-IT-TIMI-Studie zufolge gilt das jedoch nur mit Einschränkungen. Der Vorteil einer aggressiven Statintherapie gegenüber einer konventionell dosierten Therapie fiel bei Patienten mit akutem Koronarsyndrom umso geringer aus, je niedriger ihr Ausgangs-LDL war. In der Patientengruppe mit einem medianen LDL von $81 \mathrm{mg} / \mathrm{dl}$ war zwischen den beiden Statintherapien kein Unterschied mehr feststellbar.

J Am Coll Cardiol 2008;52:914-920

\section{STABILE KHK}

\section{Kann die PTCA doch Leben verlängern?}

Die Aufdehnung verengter Koronararterien bringt für Patienten mit stabiler KHK keinen Überlebensvorteil gegenüber einer rein medikamentösen Therapie: Das war die klare Aussage der im letzten Jahr vorgestellten COURAGE-Studie. Eine aktuelle Metaanalyse aus dem Deut-

- Quelle: Europäischer Herzkongress, München, September 2008. Einen ausführlichen Bericht lesen Sie in der nächsten MMW. schen Herzzentrum in München stellt dieses Ergebnis jedoch infrage. Bei der Auswertung von 17 Studien inklusive COURAGE ergab sich eine 20\%ige Reduktion der Gesamtmortalität durch die PTCA (271/3675 vs. 335/3838). Kritiker bemängeln jedoch, dass sehr unterschiedliche Studien, auch solche mit Postinfarktpatienten, in die Metaanalyse einbezogen wurden.

J Am Coll Cardiol 2008;52:894-904

\section{KHK MIT LV-DYSFUNKTION}

\section{Herzfrequenzen über 70 sind gefährlich}

Bei KHK-Patienten mit LV-Dysfunktion sollten Sie therapeutisch eine Herzfrequenz von unter $70 / \mathrm{min}$ anstreben. Denn bei höherer Frequenz steigt das Risiko für Herztod, Herzinfarkt und Herzinsuffizienz signifikant an, so ein Ergebnis der BEAUTIFUL-Studie.

Neben einem Betablocker kann dabei auch Ivabradin eingesetzt werden. Der If-Kanal-Blocker ist sicher bei LV-Dysfunktion und Kombination mit einem Betablocker. Ob er auch die Prognose verbessert, darauf gab die BEAUTIFUL-Studie keine klare Antwort. Hoffnung macht eine Subgruppe: Bei Patienten mit einer Herzfrequenz über $70 / \mathrm{min}$ schützte Ivabradin vor koronaren Komplikationen.

\section{ACE-HEMMER-UNVERTRÄGLICHKEIT}

\section{$\mathrm{AT}_{1}$-Blocker nur mäßig wirksame Alternative}

Wenn kardiovaskuläre Hochrisikopatienten den ACE-Hemmer Ramipril erhalten, senken sie damit ihr Risiko für Herztod, Herzinfarkt und Schlaganfall hochsignifikant um $22 \%$. Dies ist seit der HOPE-Studie bekannt. Der ACE-

Hemmer ist für diese Patienten Standard. Doch 10 bis 20\% der Patienten vertragen ihn nicht. Alternativ kann mit Telmisartan behandelt werden, wie jetzt die TRANSCEND-Studie ergab. Die Risikoreduktion für den gleichen Endpunkt lag bei $13 \%$ und war grenzgradig statistisch signifikant.

\section{PAVK-PATIENTEN ZUR GEFÄSS-OP}

\section{Nicht ohne Statin}

Patienten mit arterieller Verschlusskrankheit sollten vor einer Gefäßoperation mit einem Statin behandelt werden - auch wenn sie keine erhöhten Cholesterinwerte aufweisen. Denn dadurch wird die Rate kardialer Komplikationen halbiert. Dies zeigte die DECREASE-III-Studie. Verwendet wurde retardiertes Fluvastatin $80 \mathrm{mg} / \mathrm{d}$. Die Autoren gehen von einem plaquestabilisierenden, entzündungshemmenden Effekt aus. 